\title{
Rapid screening assay for precise and reliable estimation of cyanide content in sorghum
}

\author{
Rama Harinath Reddy ${ }^{1}$, Balasamy Jayaraman Karthikeyan ${ }^{2}$, Somanath Agasimani ${ }^{3}$, Ramadoss \\ Bharathi Raja ${ }^{2}$, Venkatesan Thiruvengadam ${ }^{2}$ and Sundaram Ganesh Ram $^{2 *}$
}

\author{
${ }^{1}$ Faculty of Veterinary and Agricultural Sciences, University of Melbourne, Dookie College, Dookie, VIC, 3647, \\ Australia \\ ${ }^{2}$ Department of Plant Genetic Resources, Centre for Plant Breeding and Genetics, Tamil Nadu Agricultural \\ University, Coimbatore - 641 003, Tamil Nadu, India \\ ${ }^{3}$ University of Agricultural Sciences, Bengaluru-560 065, Karnataka, India
}

*Corresponding author: ganeshgene@gmail.com

\begin{abstract}
Determining the cyanogenic potential of crop plants using traditional biochemical methods are laborious and time consuming. Presently, the Feigl-Anger paper method is being employed for qualitative analysis of cyanogenic potential of plant samples in a rapid and cost effective manner. To improve this technique from its status of qualitative to a more reliable semi-quantitative assay, the Feigl-Anger Densitometry (FAD) method was developed. This method measures the intensity of blue spots on the Feigl-Anger paper, after its exposure to $\mathrm{HCN}$ released from sorghum leaf tissues during a freeze thaw cycle. For colour intensity measurements, a densitometric software was employed. The densitometry results obtained from test samples and standards were compared with corresponding values obtained from quantitative spectrophotometric analysis. The results of FAD in a set of 66 germplasm lines revealed high correspondence with cyanide contents estimated through widely used spectrophotometric method of Lambert $\left(\mathrm{R}^{2}=0.9107\right)$. Further, our method was also validated with known concentrations of $\mathrm{KCN}$ standards $\left(\mathrm{R}^{2}=0.9661\right)$. Hence it was concluded that FAD can be employed to estimate HCN content in sorghum rapidly with acceptable precision in a simple lab setup. The method can be suitable for screening of large number of mutants and segregating plant populations for the selection of low hydrogen cyanide lines in breeding programmes.
\end{abstract}

Keywords: Sorghum; cyanogenesis; dhurrin; Feigl-Anger densitometry; hydrogen cyanide.

Abbreviations: FAD_Feigl-Anger Densitometry, HCN_Hydrogen cyanide, HCN-p_cyanogenic potential, LOD_Limits of detection, LOQ_Limits of quantification.

Introduction

Sorghum (Sorghum bicolor (L.) Moench) is grown worldwide in over $38.16 \mathrm{mha}$ and accounts for production of $57 \mathrm{mt}$ of grains with an average yield of $14.9 \mathrm{t} / \mathrm{ha}$ (FAO, 2012). It is the fifth major cereal crop in the world after wheat, rice, maize and barley. In the developing countries of Asia and Africa, sorghum serves as a multipurpose crop and has diverse uses including human consumption of its grains and also its stover widely used as fodder for cattle, goats and sheep. However, in spite of its nutritional benefits the cattle are restricted to feed on the sorghum at tender leaf stages, due to the presence of cyanogenic glucoside, dhurrin, and its break down products such as hydrogen cyanide $(\mathrm{HCN})$. The dhurrin is hydrolyzed as it passes into the rumen to produce the toxic HCN or prussic acid which causes death of ruminants by interfering with the ability of red corpuscles in the blood to transfer oxygen. This poses great concern in the view of food safety which may affect the nutritive value of fodder due to its toxic effect on the feeding livestock (Busk and Moller, 2002; Etuk et al., 2012).

For a better understanding of the occurrence and significance of cyanogenic glycosides, it is important to study representative samples of large plant populations from different tissues at different growth stages. Several methods to determine their content quantitatively and qualitatively in crop species have been described for various sensitivity levels and throughput requirements. However, the principle of $\mathrm{HCN}$ estimation in all these methods relies on hydrolyzing cyanogenic glucosides present in the plant tissues exogenously and trapping the evolved $\mathrm{HCN}$ gas in a solution of sodium hydroxide (Francisco and Pinotti, 2000; Haque and Bradbury, 2002; Takos et al., 2010).

Although quantitative tests are indispensable for accurate estimations, often a semi-quantitative test would provide sufficient information in a rapid manner to study large number of samples. One such kind was the discovery of Feigl-Anger test paper by Feigl and Anger (Feigl and Anger, 1966). The Feigl-Anger paper, when placed over a sample tissue and during a freeze-thaw cycle, reacts with HCN gas evolved which results in the development of blue color. Kakes (1991) modified the Feigl-Anger paper method with a purpose to carry out rapid screening assay for large number of samples using a 96 well-microtiter plate at a time. The blue spots developed in this method have to be analyzed qualitatively. However, it is difficult to discriminate plant 
samples with various levels of HCN content by this method due to the limitations in assessment of color intensity through visual observations. To improve Kakes rapid screening technique from its status of a qualitative method to a more reliable semi-quantitative assay, our present method describes the Feigl-Anger densitometry method abbreviated as FAD. In FAD method, a densitometry step to measure the intensity of blue spots developed on the Feigl-Anger paper is introduced. Through experiments employing a set of sorghum germplasm with varied cyanogenic status, it was proved that the densitometry values correspond well with $\mathrm{HCN}$ contents determined by quantitative spectrophotometric assays.

\section{Results and Discussion}

\section{Development of FAD method}

Cyanogenesis, the release of $\mathrm{HCN}$ as a defense on damage of plant tissues, is commonly found in many families of plants. Variations in cyanogenic potential observed in plant species were found to be governed by genetic, environmental and physiological factors (Jones et al., 1999). Breeding programs and transgenic approaches that aim at reducing the cyanogenesis in several crops require accurate but also rapid quantification of $\mathrm{HCN}$ content especially in tissues used for food or feed. Although quantitative tests are necessary to estimate $\mathrm{HCN}$ content, often large populations cannot be tested with such spectrophotometric methods which are often tedious and time consuming. Nevertheless, a semi quantitative method capable of discriminating various degrees of cyanogenic and acyanogenic types is ideal to downsize the population to a small reference set. Subsequently the quantitative methods can be employed to accurately quantify the reference set.

With this objective, in the present investigation, efforts were made to develop a method that could facilitate to analyze cyanogenic potential ( $\mathrm{HCN}-\mathrm{p})$ of plant samples in a rapid, reliable and cost effective manner. Suitable rapid screening protocols were previously developed for cyanide estimation in few crop species, for example, in cassava through picrate paper by Bradbury et al. (1998) and in Lotus japonicus based on Feigl-Anger test paper by Kakes (1991). These methods are faster and more convenient than the conventional analytical procedures, and also appropriate for analysis of large number of germplasm. However, Bradbury's picrate paper method has been disadvantageous over Kakes method as the alkaline picrate reaction takes longer incubation time $(24 \mathrm{~h})$ to develop the color whereas Feigl-Anger paper takes 90 minutes to develop color. Furthermore, the later method avoids the usage of toxic and hazardous chemicals like picric acid which is also an explosive.

Kakes method works based on the intensity of blue color formed due to the oxidation of tetra-base when reacted with HCN. This method is faster, capable of analyzing 96 samples in a microtiter plate at a time, more convenient than the conventional analytical procedures and sensitive enough for analysis of small quantities of HCN. The present experiment was initiated with Kakes (1991) method with 66 germplasm accessions. Formation of blue color spots corresponding to each accession with varied intensity on the Feigl-Anger paper was observed after a single freeze-thaw cycle. The intensity of spots varied from pale to deep blue color, (Fig. 1) which indicated presence of variability for cyanide content in the accessions studied. When Feigl-Anger papers were visually examined, we experienced difficulty in differentiating accessions with slight differences in the color intensity. This hampered us to form frequency classes among accessions studied which at times led to failures in detecting resourceful accessions.

To overcome this problem, we introduced a densitometry assessment in the above method to aid in quantitative measurement of the intensity of the color spots corresponding to each sample in the Fiegl-Anger paper. After the spot development, the image of Feigl-Anger test papers was digitized immediately after $30 \mathrm{~min}$ of incubation and a numeric optical value for each spot was obtained using the densitometry software MCID analysis ver. 7.0. The intensity of blue color developed in the test paper due to release of $\mathrm{HCN}$ were deduced as relative numerical values for measure of HCN content for each accession. The range of densitometric values clearly indicated the presence of wide variation for cyanide content among the accessions studied, thereby demonstrating the feasibility of isolating low and acyanogenic accessions by this method (Table 1 and Fig. 1). This method was named as Feigl-Anger Densitometry method (FAD).

The FAD method was validated with $\mathrm{KCN}$ standard solutions of known concentrations. The different concentrations of $\mathrm{KCN}$ were prepared and tested as described in the materials and methods. It was observed that the intensity of blue color spots gradually increased with increase in the concentration of KCN standard (Fig. 2a) and a perfect linear relationship $\left(\mathrm{R}^{2}=0.9661\right)$ was shown by $\mathrm{FAD}$ standard graph (Fig. 2b).

\section{Validation of FAD by colorimetric method}

In order to further validate the proposed rapid FAD assay, the spectrophotometric method proposed by Takos et al. (2010) was followed with the same 66 accessions with suitable modifications to meet the requirements for efficient release of HCN from sorghum leaf tissues. In this study, MES buffer was replaced by distilled water and a bead beater tissue lyser was used to augment the process of lysing the leaf material, followed by a single freeze-thaw cycle instead of three freeze-thaw cycles. It was observed that continuous freezethaw cycles resulted in the escape of gaseous $\mathrm{HCN}$ which led to poor quantification in sorghum. Therefore, the freeze-thaw cycle was limited to one occasion in the present experiment, followed by addition of $\mathrm{NaOH}$ immediately after freezing to trap $\mathrm{HCN}$ and sequential addition of the reagents as described above led to color formation. The results obtained for all 66 accessions were well in accordance with the densitometry reads of FAD method $\left(\mathrm{R}^{2}=0.9107\right)$ as perfect linear relationship was found between both methods (Fig. 3).

\section{Materials and Methods}

\section{Plant materials}

A total of 66 sorghum germplasm accessions, obtained from Ramiah Gene Bank, Department of Plant Genetic Resources, center for Plant Breeding and Genetics, Tamil Nadu Agricultural University, Coimbatore was used for the present study. All 66 accessions were sown in three replications in seedling trays for collection of leaf samples on $8^{\text {th }}$ day after sowing for HCN assays.

\section{Feigl-Anger Densitometry method (FAD)}

The proposed FAD method, as modified from Feigl and Anger (1966) and Kakes (1991) methods, was tested to screen the sorghum germplasm accessions. The protocol involved the use of Feigl-Anger paper for the detection of 
Table 1. HCN concentration and FAD values of 66 sorghum accessions as estimated using colorimetry and FAD method, respectively.

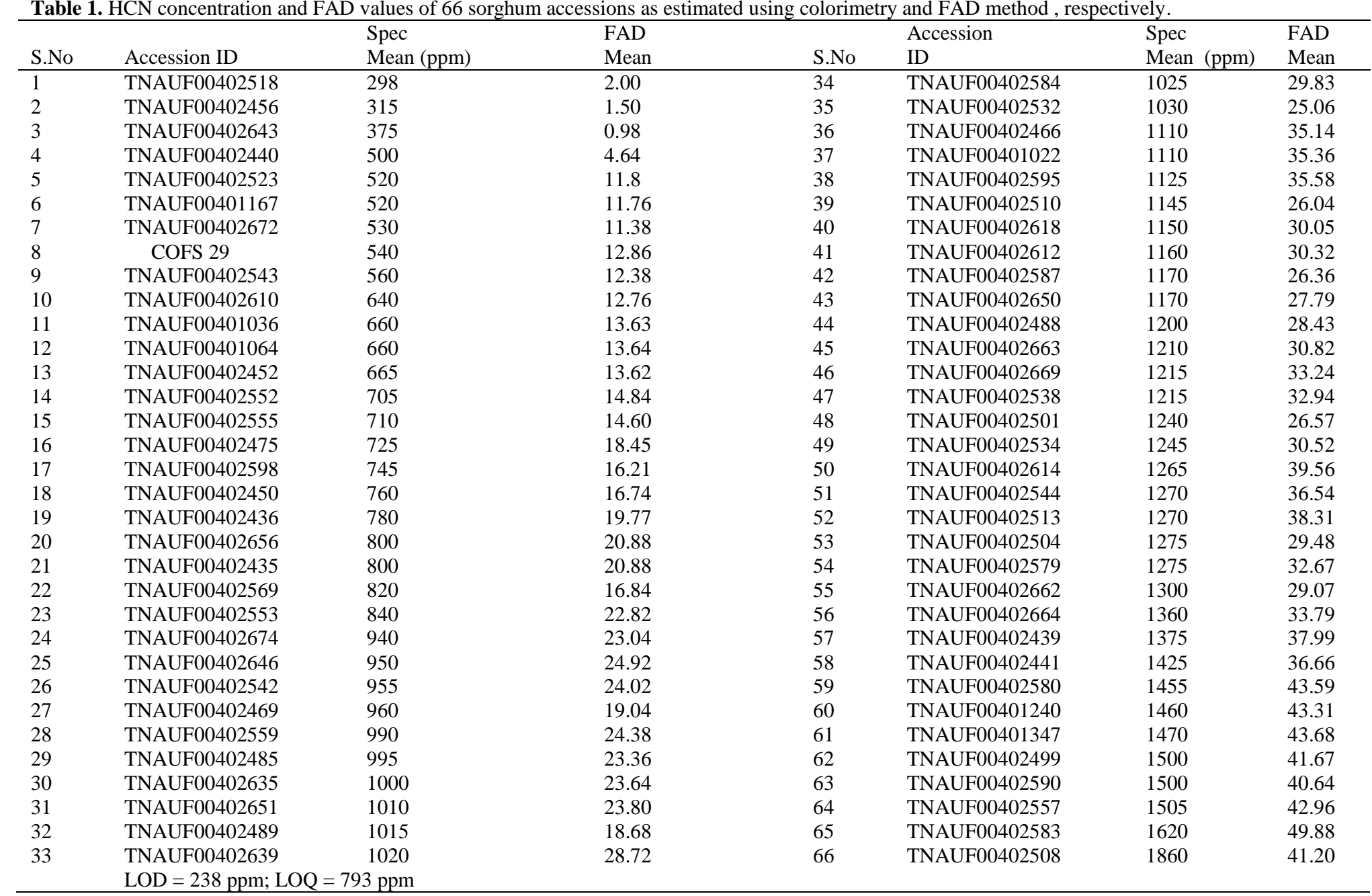

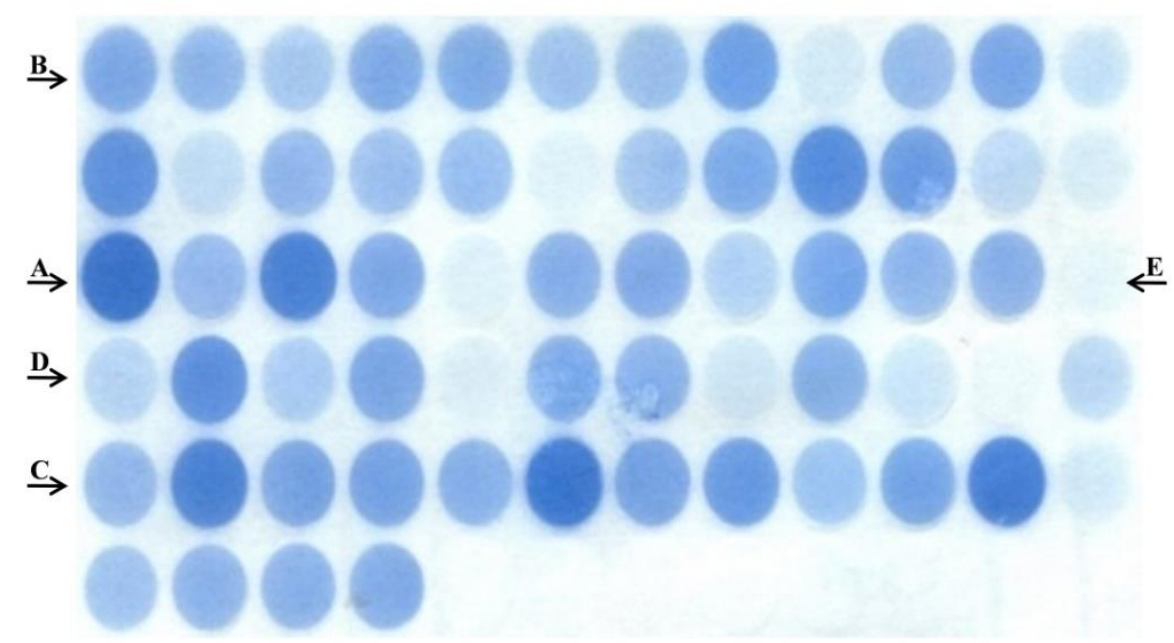

Fig 1. High throughput screening of sorghum germplasm accessions for cyanide content using Feigl-Anger paper. The accessions were discriminated for cyanide content on the basis of variation in the intensity of blue colur development. A. Very high cyanide lines, B. High cyanide lines, C. Moderate cyanide lines, D. Low cyanide lines, E. Very low cyanide lines. 
(A)

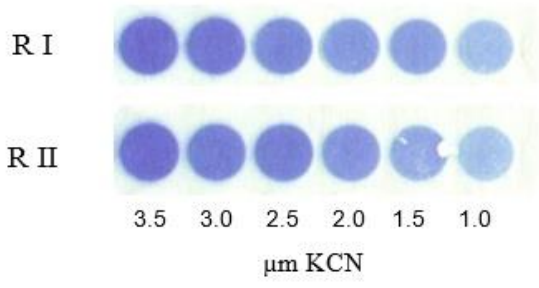

(B)

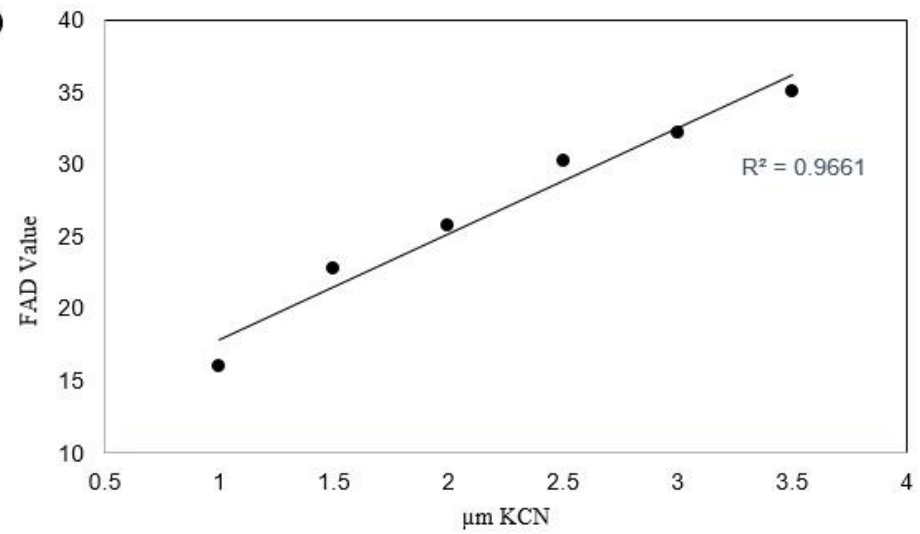

Fig 2. Validation of FAD method using $\mathrm{KCN}$ as standard. (A) Spot development on Feigl-Anger paper tested with known concentrations $(1.0,1.5,2.0,2.5,3.0$ and $3.5 \mu \mathrm{m})$ of $\mathrm{KCN}$ solution. (B) Standard curve for FAD method revealing the linear relationship.

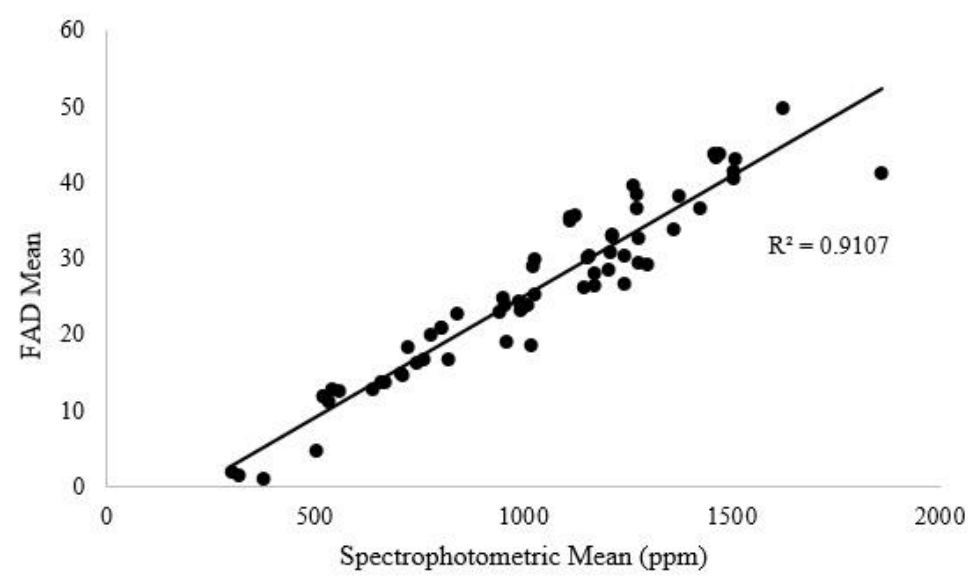

Fig 3. Regression graph showing the linear relationship for $\mathrm{HCN}-\mathrm{p}$ in leaf samples of 66 sorghum germplasm accessions as estimated by spectrophotometry and FAD methods.

HCN. This was prepared by dipping a Whatman (1MM) filter paper in a chloroform solution containing equal volumes of $1 \%(\mathrm{w} / \mathrm{v})$ copper ethylacetoacetate and $1 \%(\mathrm{w} / \mathrm{v}) 4,4$ 'methylenebis (Sigma-Aldrich). About $50 \mathrm{mg}$ of detached apical leaf tissue of each accession was placed in a 96-well microtiter plate which was overlaid with the Fiegl-Anger paper and closed airtight with a metal plate using a pair of binding clips. The set up was frozen at $-20^{\circ} \mathrm{C}$ for $1 \mathrm{~h}$ and thawed at room temperature for 30 min to develop clear blue spots over each well. The variation in color intensity level was considered as an indicative measure of the amount of $\mathrm{HCN}$ produced by the leaf. To quantify the HCN content, the test papers were scanned with a high resolution image scanner (HP LaserJet model CP1025) and the resulting images were subjected to digital image analysis using the densitometry software MCID analysis ver.7.0. A numerical value obtained from each spot corresponding to the respective plant sample was used for discrimination of samples for cyanide content and named as FAD value after the method. The experiment was conducted in two replicates. To validate the FAD method with known standards, different concentrations $1.0,1.5,2.0,2.5,3.0$ and $3.5 \mu \mathrm{M}$ of $\mathrm{KCN}$ were prepared in $0.1 \mathrm{M} \mathrm{NaOH}$ solution. An aliquot of $60 \mu \mathrm{l}$ solution of each concentration was added with $25 \mu \mathrm{l}$ of $4 \mathrm{M}$ $\mathrm{H}_{2} \mathrm{SO}_{4}$ and incubated at $-20^{\circ} \mathrm{C}$ for $1 \mathrm{~h}$. The Feigl-Anger paper was placed over the plate and thawed at room temperature for 30 min. Blue spots appeared on Feigl-Anger paper was subjected to densitometry analysis and the FAD values obtained was expressed as mean of two replicates. A standard curve was plotted with different $\mathrm{KCN}$ concentrations against the mean FAD values and regression analysis was performed to establish the linearity. 


\section{Validation of FAD method through colorimetric assay}

The quantitative spectrophotometric method as described by Lambert et al. (1975) and modified by Halkier and Moller (1989) and Takos et al. (2010) was followed for the estimation of $\mathrm{HCN}$ content in 66 sorghum germplasm accessions to validate the results of FAD method. Briefly, 50 $\mathrm{mg}$ of leaf sample ( $8^{\text {th }}$ day seedling) was weighed and transferred to a $2 \mathrm{ml}$ micro centrifuge tube. The samples were subsequently lysed in $200 \mu \mathrm{l}$ water by using a tissue disruptor and the total volume was made up to $500 \mu$ l. The samples were then subjected to single freeze-thaw cycle. To complete one freeze-thaw cycle, the samples were frozen at $-20^{\circ} \mathrm{C}$ for 1 $\mathrm{h}$ and thawed at room temperature for $30 \mathrm{~min}$. About $100 \mu \mathrm{l}$ $0.1 \mathrm{M} \mathrm{NaOH}$ was added to the samples and centrifuged at 300 rpm for $2 \mathrm{~min}$. An aliquot of $50 \mu \mathrm{l}$ was transferred from each sample to a test tube after centrifugation. Sequentially the following reagents were added to the test tube: $12 \mu \mathrm{l}$ of glacial acetic acid, $50 \mu \mathrm{l}$ of reagent A $(250 \mathrm{mg}$ succinimide and $25 \mathrm{mg} \mathrm{N}$-chloro succinimide in $100 \mathrm{ml}$ water) and $50 \mu \mathrm{l}$ of reagent $\mathrm{B}$ (a mixture of $3.5 \mathrm{ml}$ water $+1.5 \mathrm{ml}$ of pyridine + $300 \mathrm{mg}$ of barbituraic acid). After $5 \mathrm{~min}$ incubation at room temperature, the samples were scanned at $580 \mathrm{~nm}$ using a spectrophotometer. Released cyanide was calculated against standard graph of KCN standards from 400 to $2000 \mathrm{ppm}$ at intervals of $400 \mathrm{ppm}$ constituted with $0.1 \mathrm{M} \mathrm{NaOH}$.

\section{Statistical analysis}

Data on HCN contents as estimated by FAD method and the quantitative spectrophotometric method were test verified for linearity with linear regression statistics by employing XLSTAT v.7.5.2 a Microsoft excel add-on software. The limits of detection (LOD) and quantification (LOQ) were calculated using the formulae described by Rodriguez-Solana et al. (2015).

\section{Conclusion}

The results obtained show that the proposed FAD method is rapid yet accurate for quantitative screening of $\mathrm{HCN}-\mathrm{p}$ in sorghum. The currently available quantitative methods require costly equipments such as a spectrophotometer and or chromatograph which are not always affordable in the developing countries, as their acquisition and maintenance are expensive. The FAD method is economical as it does not require any sophisticated equipment or costly chemical reagents and is most suitable for crop species like sorghum which is a very important component in the human and livestock consumption in developing and under developed countries. The present study thus demonstrated that FAD method can be applied successfully for rapid quantitative screening of large number of samples as it may take only a few hours to predict the HCN-p.

\section{References}

Bradbury HJ, Hock HY, Sylvia VE (1998) Simple picrate paper kit for determination of the cyanogenic potential of cassava flour. J Sci Food Agri. 76:39-48.

Busk PK, Moller BL (2002) Dhurrin Synthesis in sorghum is regulated at the transcriptional level and induced by nitrogen fertilization in older plants. Plant Physiol. 129:1222-1231.
Etuk EB, Okeudo NJ, Esonu BO, Udedibie ABI (2012) Antinutritional factors in sorghum: chemistry, mode of action and effects on livestock and poultry. Online J Anim Feed Res. 2:113-119.

FAO (2012) Database of agricultural production Food and Agriculture Organization of the United Nation, FAOSTAT. http://faostat.fao.org/default.aspx. Accessed July 2014.

Feigl F, Anger V (1966) Replacement of benzidine by copper ethylacetoacetate and tetra base as spot-test reagent for hydrogen cyanide and cyanogen. Analyst. 91:282-284.

Francisco IA, Pinotti MHP (2000) Cyanogenic glycosides in plants. Braz Arch Biol Technol. 43: 487-492.

Halkier BA, Moller BL (1989) Biosynthesis of the cyanogenic glucoside dhurrin in seedlings of (Sorghum bicolor (L.) Moench) and partial purification of the enzyme-system involved. Plant Physiol. 90:1552-1559.

Haque MR, Bradbury JH (2002) Total cyanide determination of plants and foods using the picrate and acid hydrolysis methods. Food Chem. 77:107-114.

Jones PR, Moller BL, Hoj PB (1999) The UDP-glucose: phydroxymandelonitrile-O- glucosyltransferase that catalyzes the last step in synthesis of the cyanogenic glucoside dhurrin in Sorghum bicolor. J Biol Chem. 274:35483-35491.

Kakes P (1991) A rapid and sensitive method to detect cyanogenesis using microtiter plates. Biochem Systematics Eco. 19:519-522.

Lambert JL, Ramasamy J, Paukstelis JV (1975) Stable reagents for the colorimetric determination of cyanide by modified konig reactions. Anal Chem. 47:916918.

Rodriguez-Solana R, Salgoda JM, Dominguez JM, CortesDieguez S (2015) Comparison of soxhlet, accelerated solvent and super critical fluid extraction techniques for volatile (GC-MS and GC-FID) and phenolic compounds (HPLC-ESI/MS/MS) from Lamiaceae species. Phytochem Anal. 26:61-71.

Takos A, Lai D, Mikkelsen L, Hachem MA, Shelton D, Motawia MS, Olsen CE, Wang TL, Martin C, Rook F (2010) Genetic screening identifies cyanogenesis-deficient mutants of Lotus japonicus and reveals enzymatic specificity in hydroxynitrile glucoside metabolism. Plant Cell. 22:1605-1619. 